\title{
Clinical experience of whole-body computed tomography as the initial evaluation tool after extracorporeal cardiopulmonary resuscitation in patients of out-of-hospital cardiac arrest
}

Kelvin Jeason Yang ${ }^{1}$, Chih-Hsien Wang ${ }^{1}$, Yu-Cheng Huang ${ }^{2}$, Li-Jung Tseng ${ }^{1}$, Yih-Sharng Chen ${ }^{1 *+}$ and Hsi-Yu Yu ${ }^{1 *+}$ (D)

\begin{abstract}
Background: The routine application of whole-body CT after extracorporeal cardiopulmonary resuscitation (ECPR) in out-of-hospital cardiac arrest (OHCA) has not been extensively investigated. We aimed to evaluate the benefit of $\mathrm{CT}$ in this context.

Methods: We retrospectively analyzed all OHCA patients who had received ECPR between January 2006 to May 2019. Electronic records were reviewed to filter out patients who had a whole-body CT as their first clinical evaluation after ECPR. CT findings and major hospital outcomes were evaluated.

Results: From January 2006 to May 2019, 700 patients had received ECPR in our institution. We identified 93 OHCA patients who received whole-body $C T$ as the first clinical evaluation after ECPR. 22.6\% of those had no acute findings detected on $\mathrm{CT}$ requiring immediate treatment. In the remaining $77.4 \%, \mathrm{CT}$ had findings that might lead to alterations in clinical course. Most important findings were myocardial infarction (57.0\%), hypoxic brain injury (29.0\%), sternal/rib fractures (16.1\%), aortic dissection (7.5\%), pulmonary embolism (5.4\%), and cardiac tamponade (5.4\%). There were no significant differences in ICU/hospitalization days, time on ECMO support, survival and neurological outcomes between those with and without immediate CT. In our OHCA cohort, there were 27 patients with CT evidence of hypoxic brain injury, of whom $22.2 \%(n=2)$ managed to wean from ECMO support, $14.8 \%$ $(n=4)$ survived to discharge, but only $3.7 \%(n=1)$ survived with good neurological outcome. Hypoxic brain injury on CT has a 95\% specificity in predicting poor neurological outcome, with a false positive rate of only $3.7 \%$. Logistic regression suggested a potential correlation between $\mathrm{CT}$ findings of hypoxic brain injury and poor neurological outcome [Odds ratio $(\mathrm{OR})=12.53(1.55$ to 10.1$), p=0.02)$ ].

(Continued on next page)
\end{abstract}

\footnotetext{
* Correspondence: yschen1234@gmail.com; hsiyuyu@gmail.com

'Yih-Sharng Chen and Hsi-Yu Yu contributed equally to this work.

'Department of Surgery, National Taiwan University Hospital, College of

Medicine, National Taiwan University, No. 7, Chung-Shan South Road, Taipei

100, Taiwan

Full list of author information is available at the end of the article
}

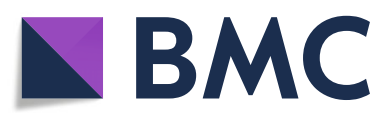

(- The Author(s). 2020 Open Access This article is licensed under a Creative Commons Attribution 4.0 International License, which permits use, sharing, adaptation, distribution and reproduction in any medium or format, as long as you give appropriate credit to the original author(s) and the source, provide a link to the Creative Commons licence, and indicate if changes were made. The images or other third party material in this article are included in the article's Creative Commons licence, unless indicated otherwise in a credit line to the material. If material is not included in the article's Creative Commons licence and your intended use is not permitted by statutory regulation or exceeds the permitted use, you will need to obtain permission directly from the copyright holder. To view a copy of this licence, visit http://creativecommons.org/licenses/by/4.0/. The Creative Commons Public Domain Dedication waiver (http://creativecommons.org/publicdomain/zero/1.0/) applies to the data made available in this article, unless otherwise stated in a credit line to the data. 
(Continued from previous page)

Conclusions: Routine whole-body CT after ECPR in OHCA patients appears to have a limited role, as the majority is caused by ACS. However, it may be a useful tool when CPR-related injury or non-ACS causes of OHCA are suspected, as well as in cases where the cause of OHCA is unknown. On the contrary, routine brain CT may be a valuable tool in guiding anticoagulant therapy during ECMO and in aiding outcome prediction.

Keywords: Extracorporeal membrane oxygenation, Cardiopulmonary resuscitation, Extracorporeal cardiopulmonary resuscitation, Out-of-hospital cardiac arrest, Computed tomography, Hypoxic brain damage

\section{Introduction}

According to the American Heart Association (AHA), there were more than 350,000 cases of out-of-hospital cardiac arrests (OHCA) in the United States in 2016, with a survival to discharge rate of only $11.4 \%$ [1]. Despite the remarkable advances in the field of resuscitation medicine, there has been limited improvement in the survival rate of OHCA patients. The use of extracorporeal membrane oxygenation (ECMO) in OHCA patient refractory to conventional cardiopulmonary resuscitation (CPR) has significantly increased the survival rate to as high as $38.7 \%$ [2-4]. The caseload of extracorporeal cardiopulmonary resuscitation (ECPR) is expected to increase continuously.

Clinical and post-mortem studies have demonstrated that acute coronary syndrome (ACS) is the most common cause of OHCA [5], however, studies have also shown that non-ACS causes account for approximately $22-34 \%$ of OHCA cases [5], which are potentially treatable if early diagnosis and treatment are achieved.

Computed tomography (CT) is a fast, cost-effective and widely available imaging modality. Recent guidelines of the European Resuscitation Council (ERC) recommend that CT scan may be performed to identify respiratory or neurological causes of OHCA, which enables prompt medical intervention [6]. However, the routine use of whole-body CT after return of spontaneous circulation (ROSC) in OHCA has not been extensively investigated and the diagnostic values remain uncertain, especially after ECPR. Over the last decade, there has been a gradual change in clinical practice in our institution. In the past, CT was mainly used as a negative selecting tool to identify those with poor prognosis (e.g. irreversible brain damage), but more recently it is used as a screening tool for other non-ACS causes for OHCA such as aortic dissection or pulmonary embolism. Therefore, this study was conducted to investigate the value of early whole-body CT after ECPR in OHCA patient.

\section{Materials and methods}

Our institution started its ECMO program in 1994 and established a task force committee for ECPR back in 2003 [3]. The task force was responsible for the collection of data and maintenance of our ECMO database. All data were prospectively collected and analyzed on a regular basis. This study was approved by the institutional review board of National Taiwan University Hospital (NTUH - 201,810,079 RIN) and the board waived the need for informed consent.

In the current study, we selectively analysed the data from our ECMO database of all adult patients (age $\geqq 18$ ) who had received ECPR following OHCA between January 2006 and May 2019. We excluded all patients whose index ECPR was performed in another institution because of uncertainty in arrest time, duration of CPR, and unstandardized ECMO management protocol. The patients whose OHCA was unwitnessed were also excluded, as well as those with incomplete follow-up data.

OHCA was defined as an event of cardiac arrest that occurred outside the hospital. For those patients who experienced OHCA, our certified emergency medical team performed CPR and defibrillation in compliance with the Advanced Cardiac Life Support guideline (ACLS), while being transported to our institution. Upon arrival at the Emergency room, the ECMO team was consulted to determine if the patient was eligible for ECPR. The contraindications, equipment and management protocol for ECMO have been described in our previously published studies $[3,4,7]$. The principle components of the ECMO circuit in our institution consisted of a centrifugal pump and a membranous oxygenator (Medtronic, Anaheim, CA, USA; Medos, Stolberg, Germany; Maquet, Rastatt, Germany). The unprimed circuit was preassembled and stored in a mobile cart. Prior to its use, the circuit was primed with normal saline containing 2 $\mathrm{U} / \mathrm{ml}$ of heparin sulfate.

Patient demographics, baseline health and initial lab data were collected immediately after the set-up of ECMO. Additional information such as low flow duration (LFD) was also recorded (defined as the interval from the start of CPR to the initiation of ECMO support). Data on major outcomes and adverse events were also collected prospectively during hospitalization. Our primary outcome was a favorable neurological outcome at discharge, which was defined as a cerebral performance category $(\mathrm{CPC})$ score of 1 or 2. 
After the set-up of ECMO circuit, the patients would then undergo the appropriate investigation to determine the cause of their arrest at the clinician's discretion. Most of the patients would either be transferred straight to the cardiac catheterization room for immediate coronary angiography (with or without primary coronary intervention) or to the radiology department for wholebody CT. For those who received immediate CT after ECPR, the radiologist would inject the contrast agent and perform the image according to a modified CT protocol based on previous studies [8-11]. The modified protocol is provided in the supplementary material (Additional file 1).

Retrospective review of the medical records was carried out to identify patients who had received wholebody contrast CT as their first investigation after ECPR. The following data were then collected: 1 . Date / time in which the CT scan was performed 2. Findings of the CT scan based on the primary radiology report. The original CT images were independently reviewed by a board certified radiologist for validation. Any discrepancy in CT findings was reconciled by a third radiologist with experience in interpreting CT images of ECMO patients. For the purpose of clarification, the diagnosis of acute myocardial infarction on CT was made when a regional myocardial perfusion defect was observed, whereas the diagnosis of hypoxic brain injury was made when features such as loss of grey-white matter differentiation and cerebral edema with effacement of cerebral sulci were present on brain CT (Additional files 2 and 3: Supplemental Figure 1 and 2). To assess the diagnostic power of CT in detecting AMI and poor neurological outcome, coronary angiographic findings and a CPC score $\geqq 3$ at discharge were used as gold standards.

\section{Statistical analysis}

Baseline patient demographics were displayed either as mean \pm standard deviation (SD) or percentage (\%). Normally distributed continuous variables were displayed as mean \pm SD and were analyzed using student $T$ test. Continuous variables with a skewed distribution were presented as median and $25-75 \%$ interquartile range and were analyzed using the Mann-Whitney $U$ test or Wilcoxon tests. Categorical variables were analyzed using the chi-square test. For those patients who received immediate $\mathrm{CT}$, logistic regression analysis was performed. Independent prognostic variables affecting the major outcome (poor neurological status at hospital discharge i.e. CPC score $3 \sim 5$ ) were first examined in univariate analysis. Variables were selected to enter the multivariable analysis if $\mathrm{P}$ value was less than 0.1 . A $\mathrm{P}$ value of $<0.05$ was considered statistically significant. The diagnostic power of whole body CT were presented as sensitivity, specificity,
True/false positive, True/false negative, positive likelihood ratio $(\mathrm{LR}+)$ and negative likelihood ratio (LR-). All statistical analysis were performed using MedCalc statistical software version 19.1 (MedCalc Software, Ostend, Belgium).

\section{Results}

Between January 2006 and May 2019, a total of 700 patients experienced cardiac arrest and received ECPR in our institution. Among them, 555 were in-hospital cardiac arrest (IHCA) patients and 9 had incomplete follow-up data, thus were excluded from our study. The remaining 136 patients who had OHCA were included and further divided into two groups depending on whether they had received whole-body CT scan as their first evaluation tool after ECPR. Ninety-three patients were classified as the immediate CT group, and the remaining 43 patients as non-immediate CT group (Fig. 1).

The baseline demographic data of the two patient groups are summarized in Table 1 . In brief, the immediate $\mathrm{CT}$ group consisted of mostly male patients and the average time interval between the set-up of ECMO to CT was $69.1 \mathrm{~min}$. Those who received immediate CT exhibited a significantly lower incidence of late stage chronic kidney disease (CKD stage $4 / 5$ ) but there was no significant difference in baseline serum creatinine level or estimated glomerular filtration rate (eGFR). In addition, the LFD in the immediate CT group was considerably longer when comparing to the non-immediate CT group $(75.4 \pm 129.9$ and $45.0 \pm 19.4 \mathrm{~min}$, respectively, $\mathrm{p}=0.03$ ). There were no significant differences in age, comorbidities, Charlson's comorbidity index (CCI), first documented rhythm and the cause of CPR between the two groups. In both groups, approximately $62 \%$ of CPR were related to acute coronary syndrome.

Among the 93 patients who received immediate CT after ECPR, 21 (22.6\%) had no acute findings requiring

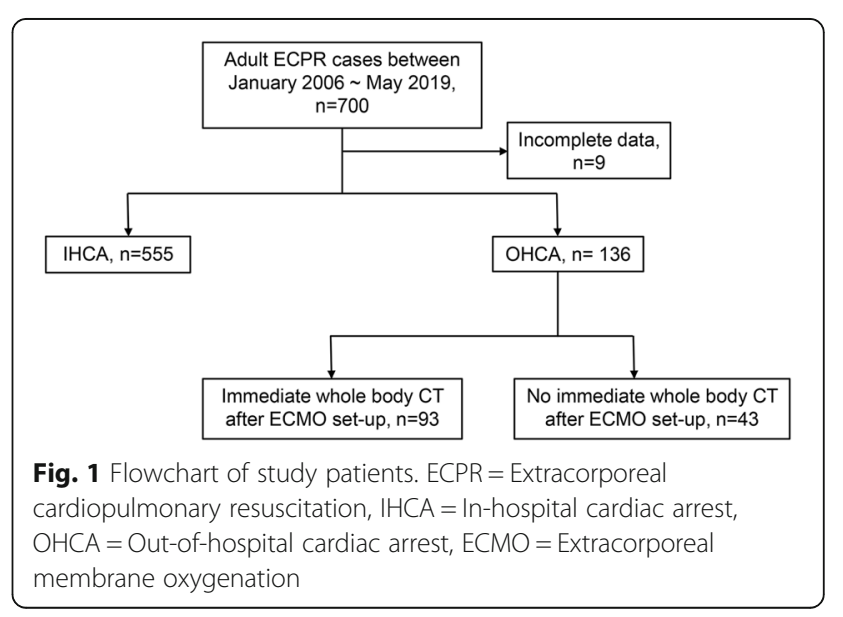


Table 1 Study population demographics

\begin{tabular}{|c|c|c|c|c|}
\hline Demographic features & All, $\boldsymbol{N}=136$ & $\begin{array}{l}\text { Immediate CT group, } \\
\boldsymbol{N}=93\end{array}$ & Non immediate CT group, $\boldsymbol{N}=43$ & $\boldsymbol{P}$ value \\
\hline Male Gender, n (\%) & $115(84.6)$ & $84(90.3)$ & $31(72.1)$ & 0.006 \\
\hline Female Gender, n (\%) & $21(15.4)$ & $9(9.7)$ & $12(27.9)$ & \\
\hline \multicolumn{5}{|l|}{ Age (year) } \\
\hline Mean \pm SD & $53.94 \pm 12.7$ & $54.7 \pm 12.2$ & $52.3 \pm 13.8$ & 0.31 \\
\hline Median (IQR) & $53.85(20.0-78.8)$ & $54.4(20-78)$ & $53.4(23-78)$ & 0.43 \\
\hline Age > 60 years, $n(\%)$ & $46(33.8)$ & $34(36.6)$ & $12(27.9)$ & 0.33 \\
\hline Age > 75 years, $n(\%)$ & $4(2.9)$ & $3(3.3)$ & $1(2.3)$ & 0.78 \\
\hline \multicolumn{5}{|l|}{ Comorbidities, n (\%) } \\
\hline Diabetes mellitus & $41(30.2)$ & $26(28.0)$ & $15(34.9)$ & 0.42 \\
\hline Hypertension & $70(51.5)$ & $47(50.5)$ & $23(53.5)$ & 0.75 \\
\hline Liver cirrhosis & $4(2.9)$ & $3(3.2)$ & $1(2.3)$ & 0.77 \\
\hline Coronary artery disease & $46(33.8)$ & $32(34.4)$ & $14(32.6)$ & 0.83 \\
\hline Peripheral arterial disease & $8(5.9)$ & $3(3.2)$ & $5(11.6)$ & 0.05 \\
\hline NYHA class 3/4 & $34(25)$ & $22(23.7)$ & $12(27.9)$ & 0.60 \\
\hline COPD & $4(2.9)$ & $1(1.1)$ & $3(7.0)$ & 0.06 \\
\hline CKD & $19(14.0)$ & $8(8.6)$ & $11(25.6)$ & $0.008^{*}$ \\
\hline CKD stage 4/ 5 & $10(7.4)$ & $4(4.3)$ & $6(14.0)$ & $0.05^{*}$ \\
\hline CKD on dialysis & $8(5.9)$ & $4(4.3)$ & $4(9.3)$ & 0.25 \\
\hline Stroke & $11(8.1)$ & $6(6.5)$ & $5(11.6)$ & 0.31 \\
\hline \multicolumn{5}{|l|}{ Causes of cardiac arrest, n (\%) } \\
\hline Acute myocardial infarction & $85(62.5)$ & $58(62.4)$ & $27(62.8)$ & 0.96 \\
\hline Chronic heart failure & $10(7.4)$ & $6(6.5)$ & $4(9.3)$ & 0.56 \\
\hline Dissecting aortic aneurysm & $4(2.9)$ & $3(3.2)$ & $1(2.3)$ & 0.77 \\
\hline Cerebral hemorrhage & $2(1.5)$ & $2(2.2)$ & $0(0)$ & 0.33 \\
\hline Pulmonary embolism & $3(2.2)$ & $3(3.2)$ & $0(0)$ & 0.24 \\
\hline Arrhythmias & $9(6.6)$ & $6(6.5)$ & $3(7.0)$ & 0.91 \\
\hline Acute myocarditis & $2(1.5)$ & $0(0)$ & $2(4.7)$ & 0.04 \\
\hline Cardiac tamponade & $1(0.7)$ & $1(1.1)$ & $0(0)$ & 0.50 \\
\hline Respiratory failure & $1(0.7)$ & $1(1.1)$ & $0(0)$ & 0.50 \\
\hline Others & $19(14.0)$ & $13(14.0)$ & $6(14.0)$ & 1.00 \\
\hline \multicolumn{5}{|l|}{ Initial Lab data (mean \pm SD) } \\
\hline Creatinine (mg/dL) & $1.96 \pm 2.08$ & $1.92 \pm 1.81$ & $2.06 \pm 2.60$ & 0.73 \\
\hline eGFR $\left(\mathrm{mL} / \mathrm{min} / 1.73 \mathrm{~m}^{2}\right)$ & $68.7 \pm 41.7$ & $64.7 \pm 30.7$ & $77.7 \pm 59.3$ & 0.10 \\
\hline Lactate (mmol/L) & $13.0 \pm 5.76$ & $12.9 \pm 5.72$ & $13.3 \pm 5.94$ & 0.74 \\
\hline \multicolumn{5}{|c|}{ Charlson's Comorbidity Index (CCI) } \\
\hline Mean \pm SD & $3.94 \pm 2.71$ & $3.68 \pm 2.55$ & $4.51 \pm 3.02$ & 0.10 \\
\hline Median (IQR) & $3.0(2.0-5.0)$ & $3.0(2.0-5.0)$ & $4.0(3.0-6.0)$ & 0.15 \\
\hline \multicolumn{5}{|c|}{ Low flow duration (min) } \\
\hline Mean \pm SD & $65.9 \pm 108.7$ & $75.4 \pm 129.9$ & $45.4 \pm 19.3$ & $0.03^{*}$ \\
\hline Median (IQR) & $49.5(38.0-60.0)$ & $53(41.0-62.5)$ & $42.0(32.0-54.3)$ & $0.004^{*}$ \\
\hline
\end{tabular}

First Documented Rhythm, n (\%) 
Table 1 Study population demographics (Continued)

\begin{tabular}{llll}
\hline Demographic features & All, $\boldsymbol{N}=136$ & $\begin{array}{l}\text { Immediate CT group, } \\
\boldsymbol{N}=93\end{array}$ & Non immediate CT group, $\boldsymbol{N}=43$ \\
\hline PEA / Asystole & $46(33.8)$ & $32(34.4)$ & $14(32.6)$ \\
ECMO to CT time (min) & & N/A value \\
Mean \pm SD & N/A & $69.1 \pm 24.84$ & N/A \\
Median (IQR) & N/A & $65.0(53.99$ to 82.24) & N/A \\
\hline
\end{tabular}

SD standard deviation, IQR Interquartile range, NYHA New York Heart Association, COPD Chronic obstructive pulmonary disease, CKD Chronic kidney disease, eGFR estimated glomerular filtration rate, VT Ventricular tachycardia, VF Ventricular fibrillation, PEA pulseless electrical activity

immediate treatment. On the other hand, 72 (77.4\%) had findings that might result in alterations in clinical course. The major acute CT findings are displayed in Table 2. The most common finding was AMI, which was found in almost up to $60 \%$ of our patient. However, there were other diagnosis other than AMI that might require medical attention or even immediate intervention, including hypoxic brain injury (29\%), acute aortic dissection (7.5\%), pulmonary embolism (5.4\%), cerebral hemorrhage/infarction (8.6\%), pneumothorax (9.7\%), cardiac tamponade (5.4\%), and sternal/rib fractures (16.1\%).

For patients who had findings of AMI on CT, the diagnostic accuracy was evaluated by correlating the results of subsequent coronary angiography, the results are displayed in Table 3. Of the 53 patients who had findings of regional myocardial perfusion defect on CT, 47/53 (88.7\%) had compatible coronary lesions confirmed by coronary angiography. Whereas for those who had no regional myocardial perfusion defect on CT, 29/40 (72.5\%) had normal coronary angiography. The sensitivity and specificity of $\mathrm{CT}$ in detecting possible AMI were 81.0 and $82.9 \%$, respectively. The LR+ was 4.7 and LRwas 0.229 .

Table 2 Findings of whole-body computed tomography performed after extracorporeal cardiopulmonary resuscitation in out-of-hospital cardiac arrest patients

\begin{tabular}{ll}
\hline $\boldsymbol{N}=93$ & Incidence of CT findings \\
\hline Acute myocardial infarction & $53(57.0 \%)$ \\
Hypoxic brain & $27(29.0 \%)$ \\
Cerebral hemorrhage & $4(4.3 \%)$ \\
Cerebral Infarction & $4(4.3 \%)$ \\
Pulmonary embolism & $5(5.4 \%)$ \\
Dissecting aortic aneurysm & $7(7.5 \%)$ \\
Cardiac tamponade & $5(5.4 \%)$ \\
Pulmonary consolidation / infiltration & $77(82.8 \%)$ \\
Atelectasis & $19(20.4 \%)$ \\
Pneumothorax & $9(9.7 \%)$ \\
Pleural effusion & $19(20.4 \%)$ \\
Bowel ischemia & $5(5.4 \%)$ \\
Sternal / rib fractures & $15(16.1 \%)$ \\
\hline
\end{tabular}

As briefly mentioned above, $29 \%$ of our OHCA cohort had evidence of hypoxic brain injury on CT after ECPR. We analyzed the CPC score of our patients and correlated with their CT findings (Table 3). Of the 27 patients who had a CT showing features of hypoxic brain injury, 26/27 (96.3\%) had a poor neurological outcome (CPC 3, 4 or 5) at discharge, with a reasonably low false positive rate of only $3.7 \%$. However, when the brain CT was normal, only 21/66 (31.8\%) had a favorable neurological outcome (CPC 1 or 2) at discharge with a false negative rate of $68.2 \%$. The sensitivity and specificity of CT in detecting poor neurological outcome were 36.6 and $95.5 \%$, respectively. The positive likelihood ratio ( $\mathrm{LR}+$ ) was 8.06 and the negative likelihood ratio (LR-) was 0.657.

The major hospital outcomes of all patients are shown in Table 4. Overall, the median duration of ECMO support was 3.0 days, and $50.7 \%$ of our OHCA patients weaned off ECMO and up to $36.8 \%$ were alive at discharge. Of those who survived at discharge, $70 \%$ had a favorable neurological outcome (CPC 1 or 2). When comparing the immediate $\mathrm{CT}$ group and the nonimmediate CT group, no significant difference in major outcomes was observed.

For patients who received immediate CT after ECPR, their respective outcomes based on CT diagnosis were analyzed and are displayed separately in supplemental materials (Additional file 4: Supplemental Table 1). We found that the AMI group exhibited the highest ECMO weaning rate $(52.8 \%)$, survival rate $(39.6 \%)$ and rate of favorable neurological outcomes $(26.4 \%)$ among all groups. On the contrary, when hypoxic brain injury is present on CT, ECMO weaning rate and rate of survival at discharge were only 22.2 and $14.8 \%$, respectively. In addition, only $3.7 \%$ of the patient had favorable neurological outcomes at hospital discharge. For both cardiac tamponade and dissecting aortic aneurysm groups, there was no survival at discharge.

Risk factor analysis for predictive factors in poor neurological outcomes (CPC 3, 4 or 5) is presented in Table 5. Univariate analysis showed that high baseline serum creatinine level $(p=0.02)$, and a CT diagnosis of hypoxic brain injury $(p=0.001)$ were potential predictive risk factors for poor neurological outcome after ECPR. Whereas dialysis during hospitalization showed a trend 
Table 3 Diagnostic strength of computed tomography in detecting acute myocardial infarction and predicting poor neurological outcome

\begin{tabular}{lll}
\hline Major CT findings, $\mathrm{n}(\%)$ & Hypoxic brain injury & Acute myocardial infarction \\
\hline Number (\%) & $27 / 93(29.0 \%)$ & $53 / 93$ (57.0\%) \\
Outcome measured & Poor neurological outcome (CPC 3/4/5) & Positive coronary angiography \\
Number of patients with / without the associated outcome & $71 / 22$ & $58 / 35$ \\
True positive & $26 / 27(96.3 \%)$ & $47 / 53(88.7 \%)$ \\
False positive & $1 / 27(3.7 \%)$ & $6 / 53(11.3 \%)$ \\
True negative & $21 / 66(31.8 \%)$ & $29 / 40(72.5 \%)$ \\
False negative & $45 / 66(68.2 \%)$ & $11 / 40(27.5 \%)$ \\
Sensitivity & $26 / 71(36.6 \%)$ & $47 / 58(81.0 \%)$ \\
Specificity & $21 / 22(95.5 \%)$ & $29 / 35(82.9 \%)$ \\
Positive likelihood ratio (LR+) & 8.06 & 4.7 \\
Negative likelihood ratio (LR-) & 0.66 & 0.229 \\
\hline
\end{tabular}

CPC cerebral performance category

of significance $(p=0.08)$. Notably, age / male gender / CCI / low flow duration / initial lactate level were not risk factors for poor neurological outcome. Results from the multi-variable logistic regression analysis revealed that CT diagnosis of hypoxic brain injury correlated with poor neurological outcome [Odds ratio $(\mathrm{OR})=12.53$ (1.55 to 10.1$), p=0.02$ ].

\section{Discussion}

Our study was initiated to evaluate the value of early diagnostic CT in OHCA patients following ECPR. Since ACS is the most common cause of OHCA, current guideline on post-resuscitation care recommends immediate cardiac catheterization if the cause of arrest was highly suspected of myocardial ischemia [12]. However, a number of clinical studies have demonstrated that a proportion of OHCA were caused by etiologies other than ACS or even mimic ACS $[5,13]$.

From our study results, we found that $77.4 \%$ of those who received immediate whole body CT had positive acute findings including dissecting aortic aneurysm, Cardiac tamponade, intracranial hemorrhage, pulmonary embolism. However, there is probably no doubt that cardiac tamponade, massive hemothorax, pleural effusion, and pneumothorax could all be detected more efficiently and with little delay by other simple image modality such as bedside ultrasonography. The rate of intracranial hemorrhage detected by CT in our study was $4.3 \%$. In other relevant literatures, the reported rate of intracranial hemorrhage in the ECMO population ranges from 10.7 to $37 \%[14,15]$. Immediate CT has the advantage of detecting intracranial bleeding early and may directly influence anti-thrombotic management during ECMO support.

When considering CPR related injury, $16.1 \%$ of the immediate CT group had findings on CT showing sternal or multiple rib fractures, which might be related to chest compression during CPR but no immediate intervention was required for those patients. Our findings seemed comparable to a French registry showing a $11 \%$ rate of chest skeletal fractures after $\mathrm{CPR}$ in OHCA patients [16]. However, in a similar study, Zotzmann et al. observed rib or sternal fractures on CT in up to $65 \%$ of their patients after ECPR [17]. Studies have shown that

Table 4 Outcomes of extracorporeal cardiopulmonary resuscitation in out-of-hospital cardiac arrest

\begin{tabular}{lllll}
\hline Outcomes & $\begin{array}{l}\text { All } \\
(\boldsymbol{n}=136)\end{array}$ & Immediate CT group $(\boldsymbol{n}=93)$ & Non-immediate CT group $(\boldsymbol{n}=43)$ & $P$ value \\
\hline Ventilator days & $11.6 \pm 18.5$ & $11.6 \pm 19.6$ & $11.7 \pm 16.2$ & $\mathrm{P}=0.98$ \\
ECMO days & $3.3 \pm 3.3$ & $3.5 \pm 3.8$ & $3.0 \pm 1.9$ & $\mathrm{P}=0.30$ \\
ICU days & $11.6 \pm 16.9$ & $10.8 \pm 12.5$ & $13.5 \pm 24.0$ & $\mathrm{P}=0.50$ \\
Hospitalization Days & $20.4 \pm 30.3$ & $19.6 \pm 28.3$ & $22.2 \pm 34.6$ & $\mathrm{P}=0.65$ \\
Die on ECMO, n (\%) & $67(49.2 \%)$ & $47(50.5 \%)$ & $20(46.5 \%)$ & $\mathrm{P}=0.66$ \\
Weaned off ECMO and die, n (\%) & $19(14.0 \%)$ & $13(14.0 \%)$ & $17(39.5 \%)$ & $\mathrm{P}=1.00$ \\
Survival on discharge, $n(\%)$ & $50(36.8 \%)$ & $33(35.5 \%)$ & $12(27.9 \%)$ & $P=0.65$ \\
CPC 1/2 on discharge, $n$ (\%) & $35(25.7 \%)$ & $23(24.7 \%)$ & $P=0.69$ \\
\hline
\end{tabular}

ECMO Extracorporeal membrane oxygenation, CPC Cerebral performance category 
Table 5 Logistic regression analysis for poor neurological outcome at discharge in patients who received early CT

\begin{tabular}{|c|c|c|c|c|}
\hline & \multicolumn{2}{|l|}{ Univariate } & \multicolumn{2}{|l|}{ Multivariate } \\
\hline & OR $(95 \% \mathrm{Cl})$ & $P$-value & $\mathrm{OR}(95 \% \mathrm{Cl})$ & $P$-value \\
\hline Age & 0.98 (0.95 to 1.03$)$ & 0.44 & & \\
\hline Age $>75$ & $1.0(0.10$ to 10.05$)$ & 0.99 & & \\
\hline $\mathrm{CCl}$ & 1.11 (0.91 to 1.36$)$ & 0.28 & & \\
\hline Male gender & $1.11(0.21$ to 5.79$)$ & 0.90 & & \\
\hline Low flow duration (min) & 1.00 (0.99 to 1.03$)$ & 0.95 & & \\
\hline Low flow duration $>60$ min & 1.27 (0.41 to 4.0$)$ & 0.68 & & \\
\hline ECMO to $\mathrm{CT}$ time (min) & 1.01 (0.99 to 1.03$)$ & 0.60 & & \\
\hline Hypoxic brain injury on $\mathrm{CT}$ & 12.4 (1.58 to 9.77$)$ & $0.001^{*}$ & $12.53(1.55$ to 10.1$)$ & $0.02^{*}$ \\
\hline Dialysis & 2.40 (0.87 to 6.61$)$ & 0.08 & 2.14 (0.72 to 6.36$)$ & 0.18 \\
\hline Initial serum creatinine & 2.22 (0.85 to 5.76$)$ & $0.02^{*}$ & 2.06 (0.72 to 5.91$)$ & 0.17 \\
\hline Initial eGFR & 0.99 (0.98 to 1.01$)$ & 0.61 & & \\
\hline Initial lactate level & $0.99(0.91$ to 1.08$)$ & 0.82 & & \\
\hline
\end{tabular}

OR Odds ratio, $\mathrm{Cl}$ Confidence interval, CCI Charlson's comorbidity index, ECMO Extracorporeal membrane oxygenation, CT computed tomography, eGFR estimated glomerular filtration rate

rib fracture is the most common injury induced by chest compression, and the prevalence may range from $13 \%$ to over 90\% [18-22]. The heterogeneous results between studies might be related to differences in methodology, study population, and detection method.

In our cohort of 93 OHCA patients who received early whole-body CT, 53 (57\%) showed a visible defect in myocardial enhancement of the corresponding coronary artery distribution. Among them, $88.7 \%$ had relevant findings on coronary angiography. The result might suggest that CT was reasonably sensitive and specific for the detection of AMI and may have a role in identifying patients who presents with atypical symptoms of ACS (such as in diabetics and in women) [23] or equivocal electrocardiographic / laboratory findings. Several case reports and retrospective studies have previously reported the detection of AMI on contrast-CT [24-27]. Gosalia et al. reported a total of 18 patients with a diagnosis of AMI, 15 of them had a focal decrease in left ventricular myocardial enhancement in a specific coronary distribution on contrast-enhanced CT [28]. The sensitivity of CT for detecting AMI in this study was approximately $83 \%$, which was comparable to our present study (88.7\%). However, since coronary angiography is almost always done for OHCA patients, we believe early CT for the detection of AMI would not be a justifiable indication. In our series, a large proportion of OHCA patients had received coronary angiography. The main reason was mainly because that the standard electrocardiographic or symptomatic criteria of AMI were often difficult to interpret in setting of $\mathrm{OHCA}$, especially when the initial rhythm was ventricular tachycardia or ventricular fibrillation. Therefore, we have a low threshold for coronary angiography in patients with risk factors for coronary artery disease in our institution.

An interesting point that worth mentioning is that from our cohort of $136 \mathrm{OHCA}$ patients, there were 43 patients who did not receive immediate CT. By reviewing the medical records, we found that 28 (65.1\%) of those who did not receive immediate CT were transported to the catheterization room for coronary angiography after ECPR. This is reasonable especially when patient presented with a typical history or risk factors for coronary artery disease. Secondly, for those who did not receive immediate CT, 15 (34.9\%) had delayed CT (mostly brain) later in their hospital admission. 9 had hypoxic brain injury, 2 had stroke, 1 had subdural hemorrhage $(\mathrm{SDH})$ and 3 had negative findings. As there is currently no local guideline or protocol to guide clinicians as to when $\mathrm{CT}$ scan should be performed after ECPR in OHCA patients in our institution, so the decision is usually made at the discretion of the clinician, taking into account of various factors such as availability, patient's clinical history, low flow duration and whether the arrest was unwitnessed / witnessed etc.

Several studies have investigated the prognostic value of brain $\mathrm{CT}$ in predicting neurological outcome following ECPR. Acute hypoxic brain injury may be manifested on brain CT as loss of Grey-white matter differentiation, brain swelling, or non-specific low density lesions [29]. More objective predictors such as grey-to-white matter ratio (GWR) [30], cortical sulcal effacement (SE), optic nerve sheath diameter (ONSD) [31] and Alberta Stroke Program Early Computed Tomography Score (ASPECTS) [32] have also been investigated. In our study, loss of grey-white matter differentiation and brain edema with effacement of cerebral sulci were mainly used to 
diagnose hypoxic brain injury, as it is less cumbersome despite being more subjective. When a CPC score of $3 \sim$ 5 were used as a measure for poor neurological outcome, we found that CT had a specificity of $95.5 \%$ for identifying patient with profound neurological impairment after ECPR, with a low false positive rate of only 3.7\%. Logistic regression analysis also suggested a potential correlation between CT findings of hypoxic brain injury and poor neurological outcome at discharge. AHA guidelines on post resuscitation arrest care had mentioned that a marked reduction in GWR on brain CT obtained with $2 \mathrm{~h}$ after OHCA may be used to predict poor outcome [33]. However, our results showed that CT had a relatively poor sensitivity in predicting poor neurological outcome. This can be explained by the fact that hypoxic brain injury may not be obvious on $\mathrm{CT}$ when it is performed early after ECPR. Based on our clinical observation, a significant proportion of our patient with normal brain appearance on initial brain CT will have findings of hypoxic brain injury when brain $\mathrm{CT}$ is repeated later in the clinical course. Based on previous literature, hypoxic brain injury is usually not apparent on brain CT immediately after cardiac arrest, but features including brain swelling and loss grey-white matter will appear after three days [34]. It is therefore reasonable to recommend that OHCA patients with normal brain CT after ECPR should have their brain CT repeated at least three days later, especially when the patient shows no sign of neurological recovery after ECPR. Our findings may have important implications for clinical decision making, especially on when to withdraw the patient from ECMO support, but one must be cautious that CT should be used as the sole indicator for the prediction of neurological outcome, mainly because of unstandardized acquisition and interpretation in most centers.

Our study has limitations. First of all, we found a significantly longer LFD in patients who received immediate $\mathrm{CT}$, and the reason for that might be that for those with relatively shorter LFD, immediate coronary angiography is often chosen as initial investigation to rule out potential occluded coronary vessels. This finding might indicate the presence of selection bias and could potentially overestimate or underestimate the incidence of the CT findings. The relatively small number and the heterogeneous nature of our study population may have had some impact on data analysis. In addition, our data was from a single institute and variation in regional practice might have some influence on the study findings. It is also possible that techniques and outcomes might have changed over time, since our study was carried out over an extended period from 2006 to 2019. Finally, from our data it was impossible to determine the temporality or the causal relationship of our CT findings. Therefore, though our data provide useful insight, larger prospective study is required to define the clinical role of CT after ECPR in OHCA patients.

\section{Conclusions}

Immediate whole-body CT after ECPR in OHCA patients remains a valuable tool despite the fact that most cases of OHCA were related to ACS. It may be particularly useful when CPR-related injury or non-ACS causes of OHCA are suspected, as well as in cases where the cause of OHCA is unknown. On the contrary, routine brain CT appears to be a valuable tool in guiding anticoagulant therapy during ECMO and in aiding outcome prediction. It seems reasonably specific, with a low false positive rate and may be considered as a predictor for poor neurological outcome in OHCA following ECPR.

\section{Supplementary information}

Supplementary information accompanies this paper at https://doi.org/10. 1186/s13049-020-00746-5.

Additional file 1. CT Protocol for Patients on Veno-arterial Extracorporeal Membrane.

Additional file 2: Figure S1. A and B, Coronal \& axial sections of contrast-enhanced computed tomography scan of the chest demonstrate regional defect in myocardial perfusion of the left ventricular wall, compatible with left main coronary lesion. C and D, Coronary angiography images of the same patient show near total occlusion of the left main coronary artery.

Additional file 3: Figure S2. A and B, CT of the brain shows loss of grey white matter differentiation, with diffuse swelling of the brain, compatible with severe hypoxic brain injury.

Additional file 4: Table S1. Outcomes of extracorporeal cardiopulmonary resuscitation in out-of-hospital cardiac arrest based on computed tomography diagnosis.

\section{Abbreviations}

ACLS: Advanced cardiac life support; ACS: Acute coronary syndrome; AHA: American heart association; AMI: Acute myocardial infarction; ASPECTS: Alberta Stroke Program Early Computed Tomography Score; CCl: Charlson's comorbidity score; Cl: Confidence interval; CKD: Chronic kidney disease; COPD: Chronic obstructive pulmonary disease; CPC: Cerebral performance category; CPR: Cardiopulmonary resuscitation; CT: Computed tomography; ECMO: Extracorporeal membrane oxygenation;

ECPR: Extracorporeal cardiopulmonary resuscitation; eGFR: estimated glomerular filtration rate; ERC: European resuscitation council; GWR: Grey-towhite matter ratio; IABP: Intra-aortic balloon pump; IHCA: In-hospital cardiac arrest; IQR: Interquartile range; LFD: Low flow duration; LR: Likelihood ratio; NYHA: New York Heart Association; OHCA: Out-of-hospital cardiac arrest; ONSD: Optic nerve sheath diameter; OR: Odds ratio; ROSC: Return of spontaneous circulation; SD: Standard deviation; SE: Sulcal effacement

\section{Acknowledgements}

We would like to acknowledge the statistical assistance provided by the Center of Statistical Consultation and Research in the Department of Medical Research, National Taiwan University Hospital.

\section{Authors' contributions}

HYY and YSC contributed to the conception and design of the study. CHW, YCH and LJT were responsible for the collection of data. KJY was responsible for the analysis of clinical data and preliminary statistical analysis. KJY and HYY have drafted the manuscript. KJY, CHW, YCH, LJT, YSC and HYY have read, revised and approved the final version of the manuscript. 


\section{Funding}

This research did not receive any specific grant from funding agencies in the public, commercial, or not-for-profit sectors.

\section{Availability of data and materials}

The dataset used and analyzed during the current study are available from the corresponding author on reasonable request.

\section{Ethics approval and consent to participate}

This study was approved by the institutional review board of National Taiwan University Hospital (NTUH - 201810079 RIN) and the board waived the need for informed consent.

\section{Consent for publication}

Not applicable.

\section{Competing interests}

The authors declare that they have no competing interests.

\section{Author details}

'Department of Surgery, National Taiwan University Hospital, College of Medicine, National Taiwan University, No. 7, Chung-Shan South Road, Taipel 100, Taiwan. ${ }^{2}$ Department of Radiology, National Taiwan University Hospital, College of Medicine, National Taiwan University, Taipei, Taiwan.

\section{Received: 23 April 2020 Accepted: 27 May 2020}

Published online: 11 June 2020

\section{References}

1. Benjamin EJ, Virani SS, Callaway CW, et al. Heart disease and stroke Statistics-2018 update: a report from the American Heart Association. Circulation. 2018;137:e67-e492.

2. Thiagarajan RR, Barbaro RP, Rycus PT, et al. Extracorporeal life support organization registry international report 2016. ASAIO J. 2017;63:60-7.

3. Chen YS, Lin JW, Yu HY, et al. Cardiopulmonary resuscitation with assisted extracorporeal life-support versus conventional cardiopulmonary resuscitation in adults with in-hospital cardiac arrest: an observational study and propensity analysis. Lancet. 2008;372:554-61.

4. Yu HY, Wang $\mathrm{CH}$, Chi NH, et al. Effect of interplay between age and lowflow duration on neurologic outcomes of extracorporeal cardiopulmonary resuscitation. Intensive Care Med. 2019;45:44-54

5. Chelly J, Mongardon N, Dumas F, et al. Benefit of an early and systematic imaging procedure after cardiac arrest: insights from the PROCAT (Parisian region out of hospital cardiac arrest) registry. Resuscitation. 2012;83:1444-50.

6. Nolan JP, Soar J, Cariou A, et al. European resuscitation council and European Society of Intensive Care Medicine Guidelines for postresuscitation care 2015: section 5 of the European resuscitation council guidelines for resuscitation 2015. Resuscitation. 2015;95:202-22.

7. Wang $\mathrm{CH}$, Chou NK, Becker LB, et al. Improved outcome of extracorporeal cardiopulmonary resuscitation for out-of-hospital cardiac arrest--a comparison with that for extracorporeal rescue for in-hospital cardiac arrest. Resuscitation. 2014:85:1219-24

8. Acharya J, Rajamohan AG, Skalski MR, Law M, Kim P, Gibbs W. CT angiography of the head in extracorporeal membrane oxygenation. AJNR Am J Neuroradiol. 2017;38:773-6.

9. Lee $\mathrm{S}$, Chaturvedi A. Imaging adults on extracorporeal membrane oxygenation (ECMO). Insights Imaging. 2014;5:731-42.

10. Lidegran MK, Ringertz HG, Frenckner BP, Lindén VB. Chest and abdominal $C T$ during extracorporeal membrane oxygenation: clinical benefits in diagnosis and treatment. Acad Radiol. 2005;12:276-85.

11. Liu KL, Wang YF, Chang YC, et al. Multislice CT scans in patients on extracorporeal membrane oxygenation: emphasis on hemodynamic changes and imaging pitfalls. Korean J Radiol. 2014;15:322-9.

12. Deakin CD, Morrison LJ, Morley PT, et al. Part 8: advanced life support: 2010 international consensus on cardiopulmonary resuscitation and emergency cardiovascular care science with treatment recommendations. Resuscitation. 2010;81(Suppl 1):e93-e174.

13. Kim YJ, Min SY, Lee DH, et al. The role of post-resuscitation electrocardiogram in patients with ST-segment changes in the immediate post-cardiac arrest period. JACC Cardiovasc Interv. 2017;10:451-9.
14. Lidegran $M$, Palmer $K$, Jorulf $H$, Lindén $V$. CT in the evaluation of patients on ECMO due to acute respiratory failure. Pediatr Radiol. 2002:32:567-74.

15. Lidegran MK, Mosskin M, Ringertz HG, Frenckner BP, Lindén VB. Cranial CT for diagnosis of intracranial complications in adult and pediatric patients during ECMO: clinical benefits in diagnosis and treatment. Acad Radiol. 2007:14:62-71.

16. Champigneulle B, Haruel PA, Pirracchio R, et al. Major traumatic complications after out-of-hospital cardiac arrest: insights from the Parisian registry. Resuscitation. 2018;128:70-5.

17. Zotzmann V, Rilinger J, Lang CN, et al. Early full-body computed tomography in patients after extracorporeal cardiopulmonary resuscitation (eCPR). Resuscitation. 2020;146:149-54.

18. Sampson MA, Colquhoun KB, Hennessy NL. Computed tomography whole body imaging in multi-trauma: 7 years experience. Clin Radiol. 2006;61:3659.

19. Lardi C, Egger C, Larribau R, Niquille M, Mangin P, Fracasso T. Traumatic injuries after mechanical cardiopulmonary resuscitation (LUCAS2): a forensic autopsy study. Int J Legal Med. 2015;129:1035-42.

20. Smekal D, Lindgren E, Sandler H, Johansson J, Rubertsson S. CPR-related injuries after manual or mechanical chest compressions with the LUCAS device: a multicentre study of victims after unsuccessful resuscitation. Resuscitation. 2014:85:1708-12.

21. Buschmann CT, Tsokos M. Frequent and rare complications of resuscitation attempts. Intensive Care Med. 2009;35:397-404.

22. Hoke RS, Chamberlain D. Skeletal chest injuries secondary to cardiopulmonary resuscitation. Resuscitation. 2004:63:327-38.

23. Bedinghaus J, Leshan L, Diehr S. Coronary artery disease prevention: what's different for women? Am Fam Physician. 2001;63:1393-400.

24. Baba A, Okuyama Y, Yamazoe S, Mogami T. Acute myocardial infarction on contrast-enhanced computed tomography. Imaging J Clin Med Sciences. 2016:3:004-5.

25. Hagdrup C, Ulriksen PS, Madsen PL. Acute anterior myocardial infarction seen on conventional iodine-contrast CT. Radiol Case Rep. 2017;12:635-7.

26. Katinakis $P$, Knaapen $P, M d ~ G, A R J G$. Computed tomography to detect acute myocardial infarction. Neth J Crit Care. 2012;16:29-30.

27. Warraich HJ, Benson CC, Khosa F, Leeman DE. Diagnosis of acute myocardial infarction on computed tomography angiogram. Circulation. 2014;129:272-3.

28. Gosalia A, Haramati LB, Sheth MP, Spindola-Franco H. CT detection of acute myocardial infarction. AJR Am J Roentgenol. 2004;182:1563-6.

29. Inamasu J, Miyatake S, Nakatsukasa M, Koh H, Yagami T. Loss of gray-white matter discrimination as an early $\mathrm{CT}$ sign of brain ischemia/hypoxia in victims of asphyxial cardiac arrest. Emerg Radiol. 2011;18:295-8.

30. Lee $\mathrm{YH}, \mathrm{Oh} \mathrm{YT}$, Ahn HC, et al. The prognostic value of the grey-to-white matter ratio in cardiac arrest patients treated with extracorporeal membrane oxygenation. Resuscitation. 2016;99:50-5.

31. Ryu JA, Chung CR, Cho YH, et al. The association of findings on brain computed tomography with neurologic outcomes following extracorporeal cardiopulmonary resuscitation. Crit Care. 2017;21:15.

32. Ryu JA, Lee $\mathrm{YH}$, Chung $\mathrm{CR}$, et al. Prognostic value of computed tomography score in patients after extracorporeal cardiopulmonary resuscitation. Crit Care. 2018:22:323.

33. Callaway CW, Donnino MW, Fink EL, et al. Part 8: post-cardiac arrest care: 2015 American Heart Association guidelines update for cardiopulmonary resuscitation and emergency cardiovascular care. Circulation. 2015;132(18 Suppl 2):S465-82.

34. Zingler VC, Krumm B, Bertsch T, et al. Early prediction of neurological outcome after cardiopulmonary resuscitation: a multimodal approach combining neurobiochemical and electrophysiological investigations may provide high prognostic certainty in patients after cardiac arrest. Eur Neurol. 2003:49:79-84.

\section{Publisher's Note}

Springer Nature remains neutral with regard to jurisdictional claims in published maps and institutional affiliations. 